\title{
Studies on Humanlike Robots - Humanoid, Android and Geminoid
}

\author{
Hiroshi Ishiguro \\ Department of Adaptive Machine Systems, Osaka University \\ ATR Intelligent Robotics and Communication Laboratories \\ JST ERATO ASADA Synergistic Intelligence System Project \\ ishiguro@ams.eng.osaka-u.ac.jp \\ http://www.ed.ams.eng.osaka-u.ac.jp
}

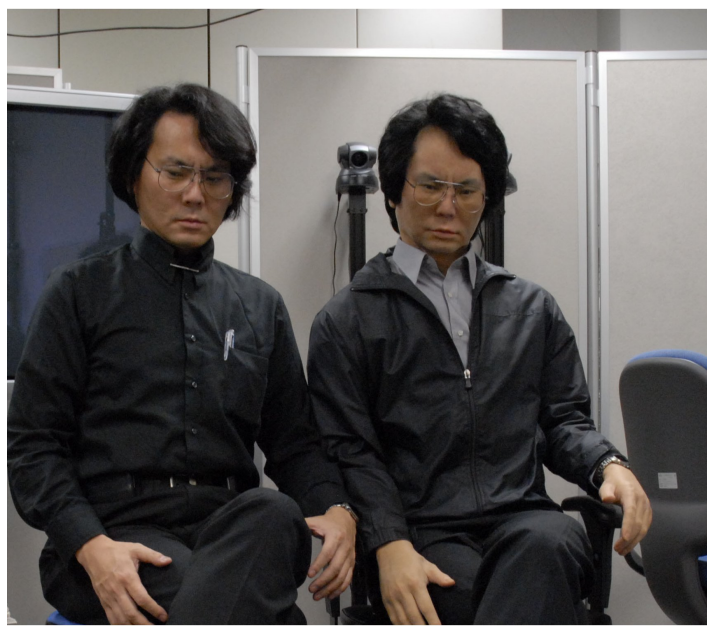

Why are we attracted to humanoids and androids? The answer is simple. We, humans, always anthropomorphize targets of interaction. In other words, we find a human itself in the humanoid. This is the reason why I am studying on humanoids and androids.

I have encountered the uncanny valley problem when I have developed the child android. Then, I have developed the female androids for compensating the problem and studied on human likeness represented with the robot in both of Robotics and Cognitive Science. However, a more serious problem is that the android could not naturally talk with people because of lack of the perfect AI. Therefore, I have developed the geminoid that is a tele-operated android connected through the Internet and studied on human-like presence.

Recently, I am focusing on the complicated mechanism of the humanlike robots and origin of the social intelligence that appears among humans and robots. This talk will introduce the series of the humanlike robot studies and discusses the fundamental issues. 